\title{
Evaluation of adherence to pediatric status epilepticus management guidelines in Saudi Arabia
}

\author{
Bashayer A. AlMohaimeed, MD, Khaled J. Hundallah, MD, Fahad A. Bashiri, MD, Sulaiman A. AlMohaimeed, MD,
} Brabim M. Tabarki, $M D$.

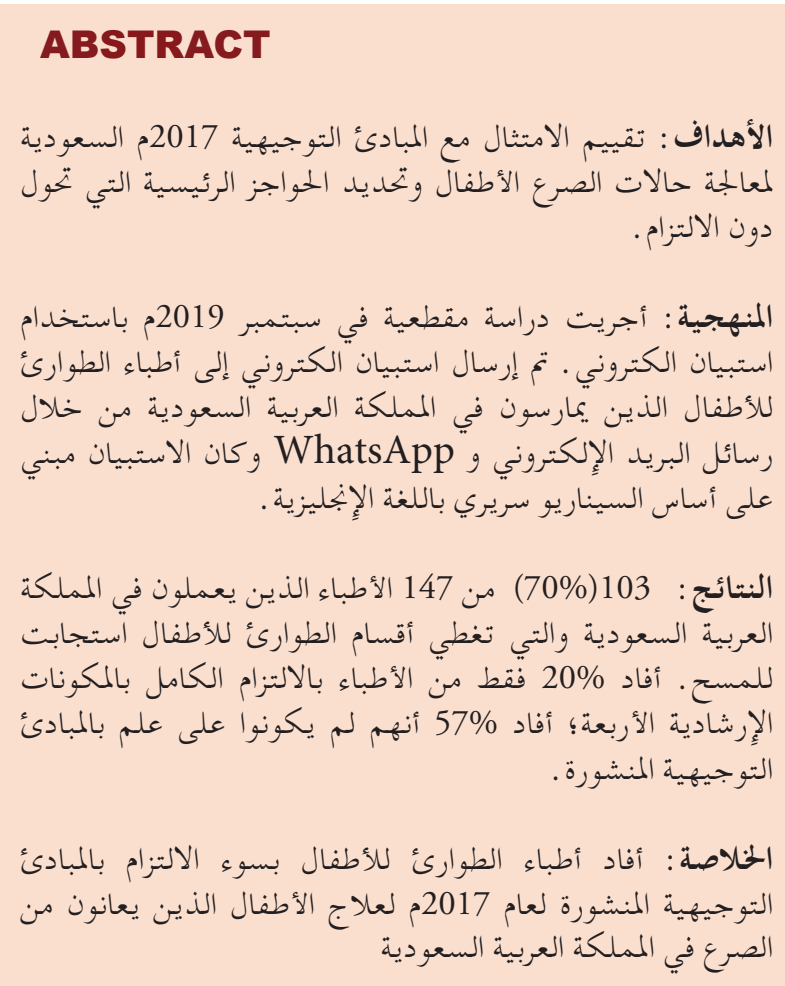

Objectives: To assess compliance with the 2017 Saudi pediatric status epilepticus management guidelines and to printout the main obstacle for adherence to the guidelines.

Methods: A Cross sectional study conducted in September 2019, using electronic survey. The survey sent to all the Pediatric Emergency physicians practicing in Kingdom of Saudi Arabia (KSA) through emails and WhatsApp and the questionnaire based on clinical scenario written in English language.

Results: One hundred and three (70\%) of 147 physicians working in KSA and covering pediatric emergency departments responded to the survey. Only $20 \%$ of the physicians reported full compliance to all 4 guideline components; $57 \%$ reported that they were not aware of the published guidelines.
Conclusion: Pediatric emergency physicians reported poor compliance to the 2017 published guidelines for the treatment of children with convulsive status epilepticus in KSA.

\section{Neurosciences 2020; Vol. 25 (3): 182-187} doi: 10.17712/nsj.2020.3.20190106

From the Division of Pediatric Neurology (AlMohaimeed B, Hundallah, Tabarki), Division of Pediatric Critical Care (AlMohaimeed S), Prince Sultan Military Medical City, and from the Division of Neurology (Bashiri), Department of Pediatrics, College of Medicine, King Khalid University Hospital, King Saud University, Riyadh, Kingdom of Saudi Arabia

\section{Received 24th November 2019. Accepted 1st March 2020.}

Address correspondence and reprint request to: Dr. Brabim Tabarki, Division of Neurology, Department of Pediatrics, Prince Sultan Military Medical City, Riyadh, Kingdom of Saudi Arabia. E-mail: btabarki@hotmail.com

ORCID ID: https://orcid.org/0000-0001-6240-0489

$\mathrm{P}$ ediatric convulsive status epilepticus (CSE) is a common emergency in neurology, which requires urgent treatment, due to the high rates of mortality and morbidity, needing early intervention to prevent neurological sequelae. ${ }^{1-2}$ The International League Against Epilepsy defines SE as "a single epileptic seizure of longer than 30-minute duration or a series of epileptic seizures during which function is not regained between ictal events in a longer than 30-minute period". ${ }^{3}$ Recently, Status epilepticus management protocols including the Neurocritical care society guidelines have adopted the operational definition of generalized convulsive status epilepticus (GCSE) as "more than 5 minutes or either continuous seizures or two or more seizures with incomplete recovery of consciousness in between" because seizures that last more than 5 minutes are likely to be prolonged. ${ }^{4}$ The incidence of SE globally is $10-58$ patients per year per 100,000 population; the frequency is estimated to be 50 per year per 100,000 population, and the mortality rate to be $2.7-22 \%$. The 
SE in children has been identified as a high priority health topic. ${ }^{5}$

Treatment of CSE has evolved over the years, with benzodiazepines being the recommended first-line treatment. ${ }^{6}$ The spaciest opinion supports draw a protocol to facilitate urgent treatment, and several studies have shown that put up guideline for status epilepticus (SE) management may improve disease management and outcome..$^{7-10}$ Nevertheless, a recent systematic review highlights that not following the guideline may lead to adverse outcomes. ${ }^{11}$

In a study carried out at Suez Canal University Hospital to Evaluation of Adherence to The Neurocritical Care Society Guidelines 2012 (NCS) in the Management Status Epilepticus Cross section observational study among 81 patients presenting with generalized convulsive status epilepticus at shows: adherence to status epileptics protocol in the Emergency Department of Suez Canal University Hospital was (55.7\%) and adherence for Emergent AEDs therapy was $(28.4 \%)$. $^{5}$

In another study done in Rwanda, cross-sectional, Data were collected by interviewing 213 nurses and doctors working in 11 hospitals around Southern Province of Rwanda, using a questionnaire based on clinical situations and the result shows: poor adherence to the national protocol for the management of status epileptics. ${ }^{12}$ In 2017, a clinical practical guideline for the Saudi healthcare context was developed using a guideline adaptation process to support relevant clinicians managing CSE in children. ${ }^{5}$

The objective of this study was to describe the management status epileptics in pediatric emergency, to evaluate the compliance to the 2017 Saudi guidelines for CSE management, and to identify the main barriers to adherence to these guidelines in the Kingdom of Saudi Arabia (KSA).

Methods. Study design. This was a cross sectional study by using a de-identified, electronic survey (Survey Monkey), sent through emails and WhatsApp to physicians using a questionnaire based on clinical scenario written in English. Since the study presented, no risk of harm to subjects and no procedures involved for which written consent is normally required outside of the research context, the principle of implied consent was used. Questionnaire regarding the management of

Disclosure. Authors have no conflict of interests, and the work was not supported or funded by any drug company. pediatric patients with status epileptics. The study was approved by the research review board at Prince Sultan Military Medical City, Riyadh, KSA and conducted in line with the Strengthening the Reporting of Observational Studies in Epidemiology for RespondentDriven Sampling Studies (STROBE-RDS) guidelines. ${ }^{13}$

Development of the written questionnaire. Participants were asked to describe the management of a theoretical scenario about 7-year-old boy, unknown any medical illness, brought by his parents, to the emergency room. He has generalized tonic-clonic seizures lasting for more than $5 \mathrm{~min}$. On examination, the heart rate is 137 beats $/ \mathrm{min}$, blood pressure is $98 / 75$ $\mathrm{mmHg}$, and temperature is $37^{\circ} \mathrm{C}$.

Specific questions was developed to address each item in the guidelines which contain 4 main components of the CSE management algorithm: the stabilization phase, first-line treatment with benzodiazepines, second-line therapy, third-line therapy, and referral to the intensive care unit (ICU); they were also asked about the investigations that they would take for this patient and the typical antiepileptic medications they would use.

Study population. There were 147 eligible pediatric emergency physicians identified from the Saudi emergency society database, We choose to send the survey to all pediatric emergency physician directly and not only the chairmen in order to decrease population selection bias. The primary target that at least 20\% of each region around Saudi Arabia of the emergency physicians involved in the management of SE would participate in the study.

Evaluation will be made according the 2017 Saudi guidelines for CSE management. On September 1, 2019, the survey was sent out through e-mail or whats app to the eligible participants. To improve the response rate and increase the study size a reminder was sent every month, until the closure of the study on October 31, 2019.

Inclusion and exclusion: All physician covering pediatric ER with title: consultant or specialists (boardcertified) were included in the study. However, resident, fellowes were excluded from the study.

Statistical analysis. Categorical data were expressed as frequencies (\%). Categorical data were expressed as frequencies (\%). Compliance with guidelines was determined for the 4 main components of the 2017 Saudi guidelines for CSE management. The management of the CSE algorithm: the stabilization phase, first-line treatment with benzodiazepines, second-line therapy, third-line therapy, and referral to the intensive care unit (ICU). Data sets were analyzed for the current study were available from the corresponding author upon 


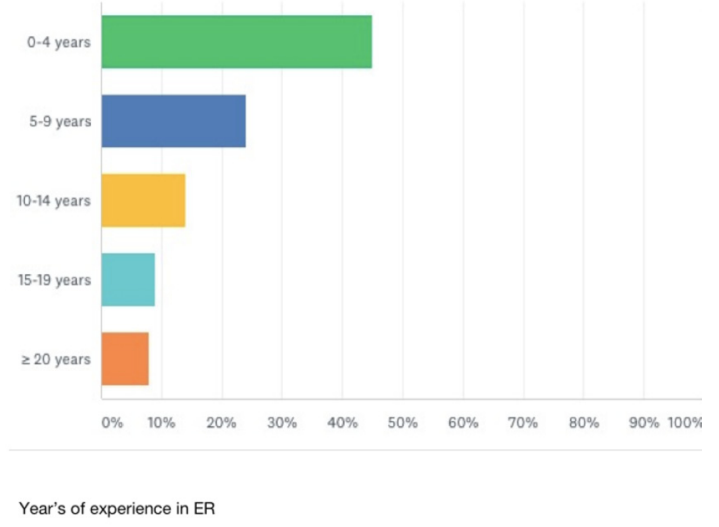

Figure 1- Shows the year's of experience in ER from 147 Pediatric Emergency Physicians in Saudi Arabia.

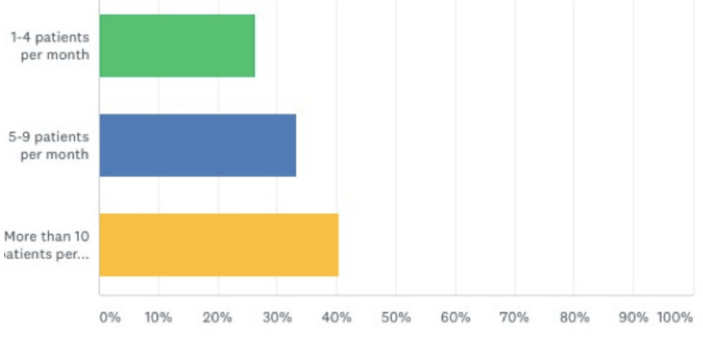

Number of patients per month

Figure 2 - Number of patients with seizures per month from the Pediatric Emergency Physicians in Saudi Arabia.

request.

Results. Respondent demographics. Out of 147 eligible pediatric emergency physicians working in Saudi Arabia, $103(70 \%)$ responded to the questionnaire. Approximately $70 \%$ had experience for less than 10 years as pediatric emergency physicians: $45.63 \%$ for < 5 years, $24.27 \%$ for $5-9$ years, $13.59 \%$ for $10-14$ years, and $16.51 \%$ for more than 15 years (Figure 1).

Seven percent responded from private hospitals, while $93 \%$ responded from government hospitals, including 34\% from academic teaching, 39\% from nonacademic teaching, and $20 \%$ from non-teaching government hospitals.

All the regions of Saudi Arabia participated in the study. Forty-one point seventy-five percent of the responders were consultants, and $58.25 \%$ were specialists. Twenty percent of the responders were adult physicians covering pediatric emergency department.

Exposure. Most of the responders (72.55\%) were dealing with more than 5 patients with seizures per

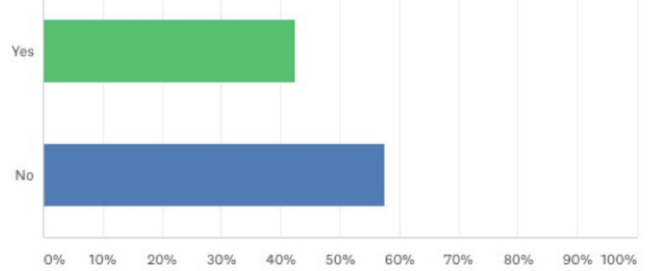

Awareness about the SE guidelines

Figure 3 - Awareness of 2017 SE guidelines from the Pediatric Emergency Physicians in Saudi Arabia.

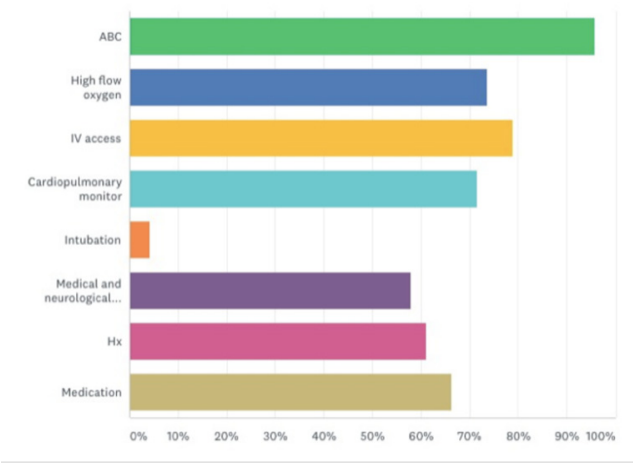

Stabilization phase

Figure 4 - Shows the stabilization phase used from the respondents

month (Figure 2). Guideline awareness and overall compliance with the guidelines. Fifty-seven percent of responders were not aware of the 2017 published guidelines for CSE management. (Figure 3)

Only $20 \%$ of the physicians showed full adherence to all guideline components.

Stabilization phase. Almost all respondents (96\%) start with $\mathrm{ABC}$ (airway, breathing and circulation), then prepare intravenous (IV) access (78\%), and provide high flow oxygen (73\%). Regarding the initial investigation to be requested, almost all responders (98\%) start with the blood glucose level test, followed by serum electrolyte (94\%) and complete blood count (76.5\%) tests. (Figure 4)

First-line treatment. If no IV access is available, all responders administer a benzodiazepine as the first-line agent. In case of available IV access, $6 \%$ of the responders prefer to start with a non-benzodiazepine agent. Twenty-three percent of the responders under-dose, administering suboptimal weight-based dosing of benzodiazepines. A common violation of the protocol administer more than 2 doses of benzodiazepines (rather than the recommendation to 


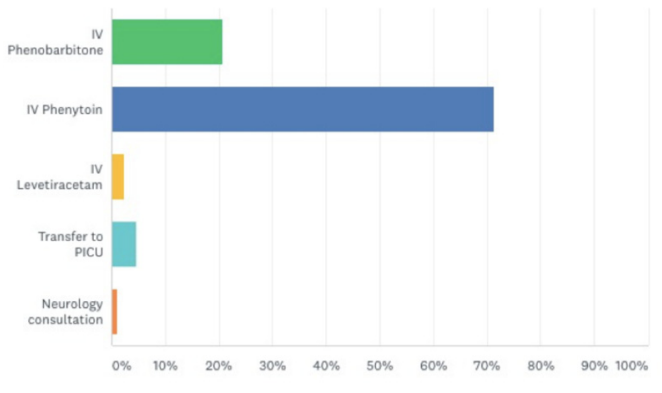

Second line treatmen

Figure 5 - Second line treatment used by the respondents.

escalate to second-line treatment), which was reported by $41 \%$ of the responders.

Regarding the timing between doses of medication, $55 \%$ prefer to wait 10 mins or less, $39 \%$ will go to the next step immediately in case of no response, and 6\% prefer to wait more than 10 mins.

Second-line treatment. The majority of the responders $(71 \%)$ prefer to use IV phenytoin, 21\% prefer IV phenobarbitone, $5 \%$ prefer to transfer the patient to the ICU, $2 \%$ prefer IV levetiracetam, and $1 \%$ contact neurology. (Figure 5)

Regarding the timing between doses of medication, $48 \%$ preferred to wait 10 mins or less, $34 \%$ go to the next step immediately if no response; and $16 \%$ prefer to wait more than $10 \mathrm{~min}$.

Regarding phenobarbital doses and infusion rates, the majority of the responders $(79 \%)$ chose the correct answer which is ( $20 \mathrm{mg} / \mathrm{kg} /$ dose; $\max 1000 \mathrm{mg} /$ dose); for infusion rate, $59 \%$ of the responders chose $(1 \mathrm{mg} /$ $\mathrm{kg} / \mathrm{min})$. Regarding phenytoin infusion rates, $62 \%$ of the responders chose the correct answer $(1 \mathrm{mg} / \mathrm{kg} / \mathrm{min})$.

Third-line treatment. In the scenario described in the questionnaire, for third-line treatment, $62 \%$ prefer at this stage to transfer the patient to the pediatric ICU, $18 \%$ decided to administer further antiepileptic drugs, and $20 \%$ preferred to seek advice from seniors.

Discussion. This study highlights a few critical points regarding the management of pediatric CSE by emergency physicians in the KSA. The findings of this survey indicate a poor compliance by the participants.

The published Saudi guidelines with regard to the stabilization phase (ABC); however, for second- and third-line treatment, several weaknesses, related to the dose of antiepileptics, infusion rate, and referral to the ICU, can be noted. We also observed significant deviations from the guidelines in first-line treatment with benzodiazepines and delays in the initiation of the second-line treatment. There is consistent underdosing of benzodiazepines during initial treatment of CSE, and often the patient receives more than 2 doses. Furthermore, if the patient has IV access, 6\% of the responders do not prescribe benzodiazepines as first-line treatment.

As known benzodiazepine is Class 1 evidence to be use as first-line therapy, this is alarming; because it results in low seizure cessation, delayed up scaling to the second-line treatment, and increase avoidable side effect like risk of respiratory depression; and higher ICU admission rates. ${ }^{9,14}$ Our findings are in line with those of previous studies regarding this matter. ${ }^{15-22} \mathrm{Administering}$ sub-optimal doses of the antiepileptics, especially benzodiazepines, is common in the management of pediatric SE. In a prospective observational study of 100 treated patients of SE requiring ICU admission, 22.5\% received benzodiazepine dose outside weight-based dose. Respiratory compromise was common in patients who received more than 2 doses of benzodiazepine or not following the weight-based dose, which show associated with an increased risk of intubation and ICU admission. ${ }^{18}$ In another retrospective study, only $32 \%$ of patients received the appropriate weight-based dose of a benzodiazepine. ${ }^{7}$ In summary, adherence to guidelines and following the recommended weight-based dosing of benzodiazepines are major targets for intervention in CSE. ${ }^{23}$

Adherence to Status epileptics protocol is the only modifiable factor in the outcome of SE. Other factors, such as age and etiology, which are strong predictors of outcome in SE, are non-modifiable. ${ }^{1,2}$ Multiple pediatric studies showed the benefits associated with the use of institutional guidelines/algorithms for the status epileptics management. In other study evaluating 47 patients with status epilepticus admitted to tertiary pediatric hospital, patients who were admitted to the ICU were more likely to received inappropriate weightbased dose than those admitted to the pediatric ward (66\% versus $26 \%$ ), and patients who were admitted to the ICU usually received more than two doses of a benzodiazepine than those admitted to the pediatric ward $(65 \%$ versus $33 \%) .{ }^{19}$ In another retrospective study, regarding management of acute seizure in pediatric patient, $61 \%$ did not receive the appropriate medications as per guidelines, most of the patients receive more than 2 doses of a benzodiazepine which result increased risk of intubation by twofold and increased risk of admission to the ICU by 1.5 -fold. ${ }^{7}$

The lack of awareness of the medical staff about the existence of the guidelines and their content is one the primary possible reasons for poor adherence to guidelines. Other barriers that may hinder the 
implementation of the national protocol could be a relatively young staff and frequent rotation of the staff assigned to emergency department. Increased awareness and training of emergency room staff and increased circulation of the published guidelines via local training could improve adherence. ${ }^{24}$

The introduction of guidelines for medical practice, a rapidly evolving and complex area, is one of many strategies to help practitioner to ensure delivery of optimal and safe care for patients. To date, the goal-patient care conformance to guidelines-has not been attained. ${ }^{25}$ Multiple studies showed that clinical guidelines are often not followed in practice, despite physicians' show acceptance and awareness of guideline-oriented, evidence-based medicine. The translation of guideline recommendations into actual medical practice is a complicated with multi-factorial process. With institutional factors, physician-related, guideline-related, and patient-related all play a role. Increased efforts should be made to implement guideline recommendations and improve guideline awareness, by using multimodal interventions such as standardized software, guidelines in print, interactive guidelines, and expert seminars. ${ }^{24,26}$

Limitation. Only consultant and Specialist were ask to participate in the questionnaire which make the sample size small.

Other limitation, the poor level of compliance to the various aspects of the SE did not exclude the presence of a perception reality gap. Implications for future research requires the increase of sample size by including ER staff, resident and ER fellow.

In conclusion, this study illustrates poor adherence to the published guidelines for the management of pediatric CSE in the KSA, partially due to the nonawareness of the guidelines. Adherence to clinical practice guidelines is, a modifiable factor that could potentially lead to improved patient outcomes. More educational interventions are necessary to improve guidelines awareness and adherence.

Acknowledgment. The authors would like to thank all physicians who responded to the survey. We would like to thank www.elsevier.com for English language editing.

\section{References}

1. Pujar SS, Martinos MM, Cortina-Borja M, Chong WKK, De Haan M, Gillberg C, et al. Long-term prognosis after childhood convulsive status epilepticus: a prospective cohort study. Lancet Child Adolesc Health 2018; 2: 103-111.

2. Gurcharran K, Grinspan ZM. The burden of pediatric status epilepticus: Epidemiology, morbidity, mortality, and costs. Seizure 2019; 68: 3-8.
3. Hommady RH, Alrifai MT, Mubayrik OK, Alayed RS, Alsemari MA, Arumayyan A, et al. Retrospective review of pediatric status epilepticus in 116 Saudi patients: predictors of outcome. Ann Saudi Med 2017; 37: 455-460.

4. Al-Shamy, S, Hosny AO, Ismail MT, Alsekaya MS, Abdeldayem M. Evaluation of Adherence to The Neurocritical Care Society Guidelines in Management of Generalized Convulsive Status Epilepticus at The Emergency Department of Suez Canal University Hospital. Journal of Neurological Disorders 2018; 06: 3-5.

5. Bashiri FA, Hamad MH, Amer YS, Abouelkheir MM, Mohamed S, Kentab AY, et al. Management of convulsive status epilepticus in children: an adapted clinical practice guideline for pediatricians in Saudi Arabia. Neurosciences (Riyadh) 2017; 22: 146-155.

6. Glauser, T, Shinnar S, Gloss D, Alldredge B, Arya R, Bainbridge J, et al. Evidence-Based Guideline: Treatment of Convulsive Status Epilepticus in Children and Adults: Report of the Guideline Committee of the American Epilepsy Society. Epilepsy Curr 2016; 16: 48-61.

7. Siefkes HM, Holsti M, Morita D, Cook LJ, BrattonS. Seizure Treatment in Children Transported to Tertiary Care: Recommendation Adherence and Outcomes. Pediatrics 2016;138: e20161527.

8. Hill, CE, Parikh AO, Ellis C, Myers JS, Litt B. Timing is everything: Where status epilepticus treatment fails. Ann Neurol 2017; 82: 155-165.

9. Tourigny-Ruel G, Diksic D, Mok E, McGillivray D. Quality assurance evaluation of a simple linear protocol for the treatment of impending status epilepticus in a pediatric emergency department 2 years postimplementation. CJEM 2014; 16: 304-313.

10. Abend NS, Loddenkemper T. Pediatric status epilepticus management. Curr Opin Pediatr 2014; 26: 668-674.

11. Uppal P, Cardamone M, Lawson JA. Outcomes of deviation from treatment guidelines in status epilepticus: a systematic review. Seizure 2018; 58: 147-153.

12. Kaputu K, Birindabagabo J, Mafuta M, Walker T, Misson, JP. Evaluation of adherence to a convulsion management protocol for children in Rwanda. Journal of Tropical Pediatrics 2013; 60: 124-128.

13. von Elm E, Altman DG, Egger M, Pocock SJ, Gøtzsche PC, Vandenbroucke JP; STROBE Initiative. The Strengthening the Reporting of Observational Studies in Epidemiology (STROBE) statement: guidelines for reporting observational studies. J Clin Epidemiol 2008; 61: 344-349.

14. Appleton R, Macleod S, Martland T. Drug management for acute tonic-clonic convulsions including convulsive status epilepticus in children. Cochrane Database Syst Rev 2018; 1: CD001905.

15. Uppal P, Cardamone M, Webber C, Briggs N, Lawson JA. Management of status epilepticus in children prior to medical retrieval: deviations from the guidelines. I Paediatr Child Health 2019; 55:1458-1462.

16. Chin RF, Verhulst L, Neville BG, Peters MJ, Scott RC. Inappropriate emergency management of status epilepticus in children contributes to need for intensive care. $J$ Neurol Neurosurg Psychiatry 2004; 75: 1584-1588.

17. Braun J, Gau E, Revelle S, Byrne L, Kumar A. Impact of nonguideline-based treatment of status epilepticus. J Neurol Sci 2017; 382: 126-130. 
18. Tobias JD, Berkenbosch JW. Management of status epilepticus in infants and children prior to pediatric ICU admission: deviations from the current guidelines. South Med J 2008; 101: 268-272.

19. Tirupathi S, Mcmenamin JB, Webb DW. Analysis of factors influencing admission to intensive care following convulsive status epilepticus in children. Seizure 2009; 18: 630-633.

20. Gaínza-Lein M, Fernández IS, Jackson M, Abend NS, Arya R, Brenton JN. et al. Association of Time to Treatment With Short-term Outcomes for Pediatric Patients With Refractory Convulsive Status Epilepticus. JAMA Neurol 2018; 75: 410-418.

21. Kämppi L, Mustonen H, Soinila S. Analysis of the delay components in the treatment of status epilepticus. Neurocrit Care 2013; 19: 10-18.
22. Unterberger I. Status Epilepticus. Journal of Clinical Neurophysiology 2016; 33: 10-13.

23. Stredny CM, Abend NS, Loddenkemper T. Towards acute pediatric status epilepticus intervention teams: Do we need "Seizure Codes"? Seizure 2018; 58: 133-140.

24. Malakooti MR, McBride ME, Mobley B, Goldstein JL, Adler MD, McGaghie WC. Mastery of Status Epilepticus Management via Simulation-Based Learning for Pediatrics Residents. J Grad Med Educ 2015; 7: 181-186.

25. Karbach U, Schubert I, Hagemeister J, Ernstmann N, Pfaff H, Höpp HW. Physicians' Knowledge of and Compliance With Guidelines; An Exploratory Study in Cardiovascular Diseases. Dtsch Arztebl Int 2011; 108: 61-69.

26. Fischer F, Lange K, Klose K, Greiner W, Kraemer A. Barriers and Strategies in Guideline Implementation-A Scoping Review. Healthcare (Basel) 2016; 4: E36. 